\title{
The van Niel International Prize for Studies in Bacterial Systematics, awarded by The University of Queensland Awarded in 2011 to George M. Garrity
}

\author{
International Committee on Systematics of Prokaryotes
}

Correspondence:

H.-J. Busse

hans-juergen.busse@vetmeduni.

ac.at

D. P. Labeda

david.labeda@ars.usda.gov

A. Oren

orena@cc.huji.ac.il

B. J. Tindall

bti@dsmz.de

\begin{abstract}
The Senate of The University of Queensland, on the recommendation of a panel of experts of the International Committee on Systematics of Prokaryotes, is pleased to present the van Niel International Prize for Studies in Bacterial Systematics for the triennium 2009-2011 to Professor George M. Garrity in recognition of his contribution made to the field of bacterial systematics. The award, established in 1986 by Professor V. B. D. Skerman of The University of Queensland, honours the contribution of scholarship in the field of microbiology by Professor Cornelis Bernardus van Niel.
\end{abstract}

Professor George M. Garrity received his Sc. D. degree from the University of Pittsburgh Graduate School of Public Health, Pittsburgh, PA, USA, in 1980 for his studies on Legionella. In 1981, he joined the Natural Products Screening Program, Merck Research Laboratories, Rahway, NJ, USA, where he worked on the characterization of bioactive products from a variety of Actinobacteria. In 1996, he was appointed Professor of Microbiology at the Department of Microbiology and Molecular Genetics, Michigan State University, East Lansing, MI.

His work centres on the use of bioinformatics and computational biology in prokaryote systematics, the development of algorithms for the classification and identification of microorganisms and microbial products, nomenclature/annotation, data visualization and knowledge mining.

He was instrumental in developing the technology for the NamesforLife project, established to resolve the ambiguity between nomenclature and biological objects and concepts, providing a new approach to the retrieval of information from diverse sources, based upon the use of nomenclature to link content. NamesforLife models the evolution of changes in biological nomenclature and terminology, resolves instances of synonymy and homonymy, and provides mapping to the underlying concepts that can be viewed in a temporal context. Using Digital Object Identifiers, names or terms are linked to permanent unique identifiers, can provide a direct path through the literature, and link to a variety of databases and other contextually relevant services. The project has also developed a Firefox add-on that can identify taxonomic names in online articles and provide up-to-date nomenclatural and taxonomic information.
He has helped develop the 'taxonomy browser', an experimental application that demonstrates how visualization and analytical techniques drawn from the field of exploratory data analysis might help to reveal the taxonomic structure of prokaryotes. When applied to very large matrices of data derived from aligned $16 \mathrm{~S}$ rRNA gene sequences, it can reveal the hidden taxonomic structure of the source organisms. Heatmaps have proven to be the most revealing visualization technique, serving as the basis for a novel graphical user interface for accessing associated information in the literature and databases. He is also involved in the Ribosomal Database Project.

He was a member of the Bergey's Manual Trust from 1996 to 2006, serving as Editor-in-Chief of the first two volumes of the second edition of Bergey's Manual of Systematic Bacteriology. He is a founding member and Editor-in-Chief of the open-access journal Standards in Genomic Sciences, a journal primarily concerned with genomics, but also draws on systematics and the need to standardize data collection, evaluation and interpretation. As Chairman of the International Committee on Systematics of Prokaryotes, he advances the interests of bacterial and archaeal systematics on all levels.

His main contribution has been at the level of supporting and providing basic infrastructure that is so essential in coordinating and integrating the diverse sets of data that accumulate in systematics and that has become a major issue in data collection, storage and interpretation.

The above citation accompanies the prize, which will also be announced at the IUMS Bacteriology and Applied Microbiology Congress in Sapporo, Japan, 6-10 September 
2011. The International Committee on Systematics of Prokaryotes wishes to congratulate Professor George M. Garrity on being awarded the prize in recognition of his contribution to prokaryote systematics.
Citation prepared by The University of Queensland and submitted on their behalf and that of the International Committee on Systematics of Prokaryotes by H.-J. Busse, D. P. Labeda, A. Oren, and B. J. Tindall. 